Reduktion der Alkoholmenge zu vereinbaren“, berichtete Kiefer.

\section{Ambulanter Entzug unter hausärztlicher Begleitung}

Hausärzte, die einen ambulanten Entzug begleiten, können Benzodiazepine, Antiepileptika (etwa Carbamazepin), Neuroleptika (etwa Tiaprid, auch in Kombination mit Carbamazepin) oder Antidepressiva (etwa Tianeptin) verordnen, um die physischen und psychischen Entzugserscheinungen $\mathrm{zu}$ mindern. Doch sollte man die Medikamente vorsichtig einsetzen. „Denn bei Menschen mit Alkoholerkrankung wirken wir mit unseren Medikamenten unter Umständen auf Gehirne ein, die nicht so stabil sind wie die von Menschen ohne chronischen Alkoholmissbrauch", mahnte Prof. Andreas Heinz, Berlin. Wichtig ist in dieser Zeit der häufige, evtl. sogar tägliche Kurzkontakt mit den Patienten, um den Verlauf der Entzugssymptomatik beurteilen zu können.

Im Anschluss an den Entzug können Anticraving-Medikamente wie Naltrexon das Risiko für einen Rückfall verringern. Dieser neu zugelassene Opioidantagonist ist für die mehrwöchige bis -monatige Phase der Alkoholentwöhnung indiziert. Laut aktueller CochraneBewertung soll die Kurzzeitbehandlung mit Naltrexon die Wahrscheinlichkeit von Rückfällen um 36\% und das Risiko für einen Behandlungsabbruch um $28 \%$ senken.

\section{Neue Kontakte müssen her}

Begleitend zur Medikamentengabe ist immer eine psychotherapeutische Behandlung einzuleiten bzw. zu vermitteln. „Eine langfristige, psychotherapeutische Behandlung hat sich als sehr effektiv erwiesen“, bestätigte Backmund. In der Zeit der Abstinenz sollte man den Patienten darin unterstützen, sein Umfeld zu verändern, neue Kontakte (etwa zu den Anonymen Alkoholikern) zu knüpfen und soziale Hilfestellungen anzunehmen.

\title{
(1) Riesenhernie wuchs immer weiter
}

Eine riesige Hernie entwickelte ein 57-Jähriger im Bereich einer Op.-Narbe am Oberkörper. Sich deswegen ein zweites Mal unters Messer zu legen, lehnte er jedoch kategorisch ab. Lieber nutzte er seine eigenen Methoden, um den Bruch zu bändigen.

Ein massiver Vorderwandinfarkt ergab im September 2003 die Indikation zur notfallmäßig operativen Myokardrevaskularisation. Daraufhin bildete sich im Bereich der Op.Narbe eine Hernie - deren Inhalt aus Magenanteilen und Darm bestand. Aufgrund des kurz zurückliegenden Akutereignisses sollte sie jedoch erst später operativ saniert werden, berichtet Dr. Carsten Böttinger aus seiner Praxis in Bad Orb. Zur Herniotomie kam es aber nie: Aus Todesangst vor einem erneuten Eingriff ließ der Mann die Hernie "weiterwachsen" und reponierte sie lieber selbst durch Zurückbeugen des Rumpfes, Einpressen des Bruchinhalts und Fixation mittels zweckentfremdeter Bauchbandage. Kasuistik aus der Praxis von Dr. Carsten Böttinger, Bad Orb, 2010
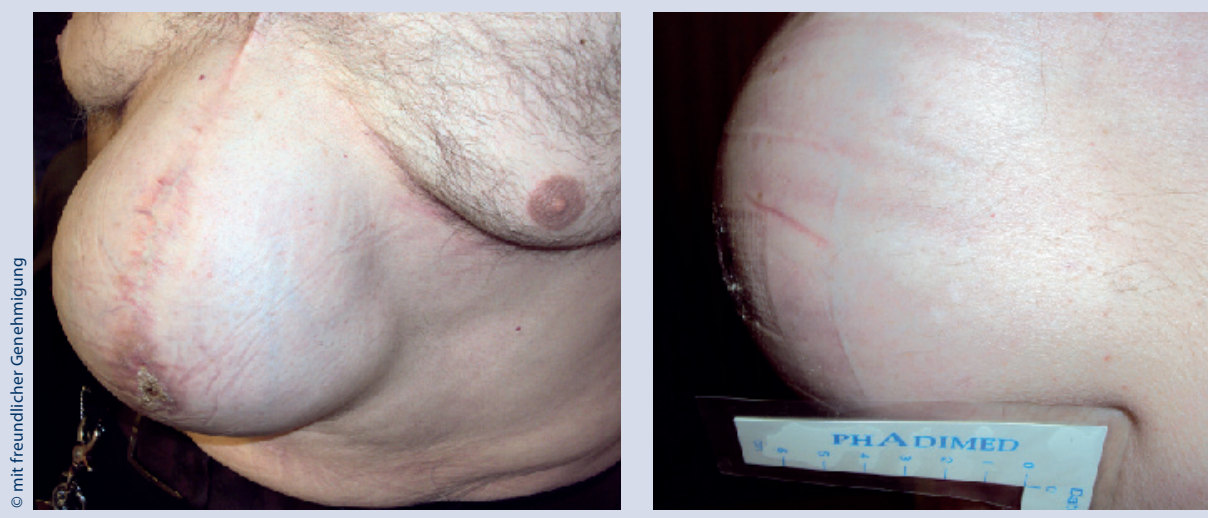

Von innen drückten Magen und Darm, von außen malträtierte ein Ulkus die Haut der Hernie.

C) Hingucker aus Praxis und Klinik

Meist geht es in der Hausarztpraxis ja eher um Schnupfen, Rückenschmerzen oder Hypertonus. Aber bestimmt läuft Ihnen auch mal der eine oder andere Patient mit einem optisch außergewöhnlichen Befund ins Haus! Machen Sie mit - schicken Sie uns einen spannenden Fall, denn wir möchten interessante Bilder aus Klinik,
Praxis und unserem großen Springer-Archiv aufgreifen und in jedem CME einen "Hingucker" bringen. Für jeden veröffentlichten Befund gibt es ein Springer Medizin Lexikon. Schicken Sie Ihren Fall an: Springer Medizin Verlag, Frau Claudia Daniels, Tiergartenstr. 17, 69121 Heidelberg, claudia.daniels@springer.com.
Mit diesem Bündel an Maßnahmen lässt sich einiges erreichen. „Dabei müssen Sie bedenken, dass Alkoholismus eine chronische Krankheit ist, die mit einem sehr desolaten körperlichen Zustand einhergeht. Aber wenn man das mit den Verläufen anderer chronischer Krankheiten vergleicht, gibt es bei Alkoholikern immerhin Phasen der Abstinenz, in denen sie zu 100\% funktionstüchtig sind und sich in den Alltag integrieren können. Ein Jahr Abstinenz ist für diese Menschen ein riesiger Gewinn“, gab Kiefer zu bedenken.
Alkoholkranke leiden überdurchschnittlich häufig zugleich unter einer psychiatrischen Störung wie Depressionen, Angststörungen oder Störungen der Impulskontrolle. Dies belegt beispielsweise eine Untersuchung an der Universität Rostock: Etwa zwei Drittel der dort behandelten Entgiftungspatienten wiesen eine komorbide psychiatrische Störung auf. Zudem beobachtete man, dass sich die Alkoholerkrankung bei Menschen mit einer Persönlichkeitsstörung früher manifestierte.

(Marion Hofmann-Aßmus) 